\title{
On a possible relationship between linguistic expertise and EEG gamma band phase synchrony
}

\author{
Susanne Reiterer ${ }^{1,2}$ *, Ernesto Pereda ${ }^{3}$ and Joydeep Bhattacharya ${ }^{4,5}$ \\ 1 Department for English Linguistics, Center for Linguistics, University of Tübingen, Germany \\ ${ }^{2}$ Department of English Studies, University of Vienna, Vienna, Austria \\ ${ }^{3}$ Department of Basic Physics, University of La Laguna, Tenerife, Spain \\ ${ }^{4}$ Department of Psychology, Goldsmiths College, University of London, London, UK \\ ${ }^{5}$ Commission for Scientific Visualization, Austrian Academy of Sciences, Vienna, Austria
}

\section{Edited by:}

Teresa Bajo, Universidad de Granada,

Spain

\section{Reviewed by:}

Steve Majerus, Université de Liège, Belgium

Alfredo Brancucci, Università 'G.d'Annunzio' di Chieti e Pescara, Italy

\section{*Correspondence:}

Susanne Reiterer, Department for English Linguistics, Center for Linguistics, University of Tübingen, Hoppe Seyler Strasse 3, 72076

Tübingen, Germany.

e-mail: susanne.reiterer@med.

uni-tuebingen.de
Recent research has shown that extensive training in and exposure to a second language can modify the language organization in the brain by causing both structural and functional changes. However it is not yet known how these changes are manifested by the dynamic brain oscillations and synchronization patterns subserving the language networks. In search for synchronization correlates of proficiency and expertise in second language acquisition, multivariate EEG signals were recorded from 44 high and low proficiency bilinguals during processing of natural language in their first and second languages. Gamma band $(30-45 \mathrm{~Hz})$ phase synchronization (PS) was calculated mainly by two recently developed methods: coarse-graining of Markov chains (estimating global phase synchrony, measuring the degree of PS between one electrode and all other electrodes), and phase lag index (PLI; estimating bivariate phase synchrony, measuring the degree of PS between a pair of electrodes). On comparing second versus first language processing, global PS by coarse-graining Markov chains indicated that processing of the second language needs significantly higher synchronization strength than first language. On comparing the proficiency groups, bivariate PS measure (i.e., PLI) revealed that during second language processing the low proficiency group showed stronger and broader network patterns than the high proficiency group, with interconnectivities between a left fronto-parietal network. Mean phase coherence analysis also indicated that the network activity was globally stronger in the low proficiency group during second language processing.

Keywords: EEG, gamma band, phase synchronization, bilinguals, second language acquisition, cortical efficiency, linguistic expertise, individual differences in proficiency

\section{INTRODUCTION}

Most brain imaging studies on bilinguals/multilinguals have been conducted with either positron emission tomography (PET) or functional magnetic resonance imaging (fMRI) with a pure emphasis on localizing brain activities (e.g., see De Bot, 2008 for review). They have not specifically investigated the functional connectivity between different and distributed brain areas, yet one of the most discussed hypotheses - the influence of second language proficiency level on the extent and distribution of brain activation - would call for a method analyzing functional cooperation and interactions of brain areas. This is frequently done in the field

\footnotetext{
Abbreviations: EEG, electroencephalography; $\mathrm{Cz}, \mathrm{C} 4$ electrode positions on scalp over central and right hemisphere areas; fMRI, functional magnetic resonance imaging; F8, electrode position on scalp (frontal right); $\mathrm{Fp}=$ frontal; $8=\mathrm{RH}$; Fp1, electrode position on scalp (prefrontal left); $\mathrm{Fp}=$ fronto-polar; $1=\mathrm{LH}$; Fp2, electrode position on scalp (prefrontal right); $\mathrm{Fp}=$ fronto-polar; $2=\mathrm{RH}$; HP, high proficiency group; HT, Hilbert transform; $\mathrm{k} \Omega$, kilo $\mathrm{Ohm} ; \mathrm{L} 1$, first language (mother tongue); L2, second language (foreign language); LH, left hemisphere; LP, low proficiency group; $\mu \mathrm{V}$, micro Volt; PET, positron emission tomography; PS (index), phase synchronization (index); RH, right hemisphere; SCA, synchronization cluster analysis; SD, standard deviation; T4, electrode position on scalp over temporal right hemisphere area; T5, electrode position on scalp over temporal left hemisphere area.
}

of EEG research by using coherence or synchronization analyses (Ward, 2003; Allefeld et al., 2005; Fries, 2005; Stam, 2005). Bilingual brain organization in terms of networks and functional cooperation has been scarcely investigated hitherto (for EEG coherence see Reiterer et al., 2005a,b; and for fMRI connectivity see Dodel et al., 2005 and Majerus et al., 2008 as examples). In fact, the study by Dodel et al. (2005) and the recent study by Majerus et al. (2008) are the only examples, to the best of our knowledge, that have investigated cortical synchronization patterns by employing fMRI connectivity analyses in bilingual language (word and sentence processing, Dodel et al., 2005) as well as native language short-term memory (STM) processing (Majerus et al., 2008). Interestingly, the first connectivity study (Dodel et al., 2005) found larger and more extended networks for the bilinguals with higher proficiency levels, contrary to many studies on bilingual fluency levels, which find fewer activated areas as a function of higher fluency levels in second languages (e.g., Perani et al., 1996, 1998; Yetkin et al., 1996; Chee et al., 2001; Hasegawa et al., 2002; Briellmann et al., 2004; Xue et al., 2004; Klein et al., 2006). The second connectivity study (Majerus et al., 2008), albeit not investigating language or bilingual language processing per se but STM processing instead, could nevertheless differentiate high from low proficiency bilinguals by 
means of fMRI connectivity patterns. They found the connectivity patterns to be characteristically diverse (rather than e.g., larger or smaller) for the two behaviorally different bilingual proficiency groups, with the low proficiency group showing a less specialized and less differentiated neural network underlying (serial order) STM processing, which, according to the authors, leads to a less efficient processing of serial order information in STM in the low proficiency group (a fact which is assumed to be causally connected to their generally poorer second language performance).

However, in the field of EEG synchronization, we did not find any comparable studies that investigated bilingual proficiency levels.

In an earlier study (Reiterer et al., 2005a), we analyzed EEG coherence in the lower and middle frequency ranges [from delta (1-4 Hz) to beta range $(13-30 \mathrm{~Hz})]$, and found a significant correlation between proficiency level and EEG coherence within the alpha band (8-12 Hz) (Reiterer et al., 2005b). The high proficiency (HP) group displayed lower coherence for both, native and foreign, language stimuli. Since the alpha band primarily reflects attentional processes, this result could possibly indicate a general language processing strategy based on general attentional processes, but not necessarily a differential language processing strategy [differentiating first (L1) from second language (L2)]. Further, the alpha band might have been too narrow to capture the differences in proficiency related to the different languages. Broad high frequency bands, such as gamma band, could be a more promising candidate to capture linguistic processes at a higher level of sophistication.

Based on these studies that revealed differences in activation patterns as a function of fluency level differences (e.g., efficient processing as in Just et al., 1996), we hypothesized that low proficiency bilinguals, as compared to high proficiency bilinguals, would be associated with a higher degree of gamma band synchronization during second language processing. Some studies (Simos et al., 2002; Micheloyannis et al., 2003; Hagoort et al., 2004; Ford et al., 2005; Weiss et al., 2005; Bastiaansen and Hagoort, 2006; Hald et al., 2006; Ihara and Kakigi, 2006; Bastiaansen et al., 2010) have already pointed to relations between gamma band synchronization and native language processing, but second language or bilingual language processing has almost not been investigated in this high frequency range. A notable exception here is the study by Ihara and Kakigi (2006), which already adverted to a putative role of the alpha and the gamma band for detecting possible differences between first and second language systems. Furthermore, we want to make a distinction between short-range or local synchronization, i.e., synchronization within a node of a functional network, and long-range synchronization, i.e., synchronization between different nodes of a network (Bastiaansen and Hagoort, 2006; Le Van Quyen and Bragin, 2007). Local gamma synchronization occurs when a large number of neurons transiently oscillate with a common phase and is primarily represented by the spectral content of the gamma band oscillation of any individual EEG electrode, whereas the long-range gamma synchronization occurs when two preferably large neuronal populations recorded by two distant EEG electrodes oscillate with a phase relationship over (at least) a few gamma oscillation cycles and is primarily represented by the degree of phase synchrony between these two EEG electrodes. The majority of the studies on native language comprehension addressed only the spectral power changes within an EEG electrode (i.e., local synchrony), while ignoring the relationship between multiple electrodes (i.e., long-range synchrony).

So, in the present work, we exclusively investigated and analyzed the long-range synchronization properties of gamma band during language comprehension in late bilinguals. To our knowledge, this is the first attempt to investigate the influence of the amount of linguistic training and expertise on long-range gamma band phase synchronization (PS).

\section{MATERIALS AND METHODS PARTICIPANTS}

We contrasted two groups of differentially proficient second language (L2 = English) speakers, who had overall comparable educational level (University students), but differed in their proficiency levels in L2 due to different amounts of training in English. The participants in the "high proficiency group" (HP) were advanced university language students studying English language and linguistics for a master's degree (last year, 5-6 years completed). Their level of English proficiency was "very good" (so-called native speaker-like performance) or "good" according to their performances at university. Additionally, this level was verified by a certified English language teacher according to oral fluency test interviews. This rating system reflected the Austrian school marking system from one to five (max-to-min) and according to this rating system the participants were divided into the following five categories "very good," "good," "medium," "lower-level," "lowestlevel." Most of the participants in the HP also studied a second foreign language (i.e., an L3) like French, Italian, or Spanish, or general linguistics. They all had high levels of linguistic training and knowledge at the time of the experiment. In other words, they have been "pre-screened" for HP at University already. As for their exposure to real life surroundings with the second language, the average amount of time they had spent abroad in an English speaking country was 10 months. The participants in the "low proficiency group" (LP) were university students of various disciplines studying for a master's degree in a subject other than language and linguistics (e.g., biology, psychology). They displayed medium to low level second language skills (corresponding to the groups "medium," "lower-level," and "lowest-level"), which were sufficient to let them pass their school leaving exams ("Matura," an equivalent to "A levels"), but since then were not developed any further. They were able to lead basic level conversations in English, but their speech was non-fluent, characterized by grammatical errors, poor pronunciation (foreign accent), slowed-down lexical access, and long pauses. The average amount of time LP participants spent abroad in an English speaking country was 5 weeks. With regard to the country where they had spent some time, the groups were homogeneous.

We strictly controlled for the variable "age of onset" of L2 learning. The average $( \pm S D)$ age of onset was 9 years ( 1 year) and was matched between the two groups. Further controlled variables were: age, handedness, gender, mother tongue, socio-educational, and cultural background, region of residence, and non-verbal and verbal IQ. Each group consisted of 22 right-handed (measured 
by the Edinburgh handedness inventory; Oldfield, 1971) female students with German as their native language. We rigidly controlled for the variable gender in order to avoid possible influences of gender onto the processing of language and its neural representations. After manual and automatized artifact control we had to exclude six subjects (mostly because of muscle artifacts and/or paroxysmal oscillations in the EEG signals) from the further statistical analyses, so that finally each group was composed of 19 participants.

Mean (SD) age was 24 years (2.3 years and 2.7 years respectively for two groups) for both groups. They were also matched for socio-cultural background and education: all participants had similar social (middle class), educational (university students), and cultural (living in Vienna) background.

The two groups differed from each other mainly in the amount of second language training they were exposed to, and the difference was approximately 6 years. Summarizing, the differences between the two groups are in their linguistic experience and knowledge, hence, in their proficiency levels in English as their L2.

The study was in compliant with the Code of Ethics of the World Medical association (Declaration of Helsinki) and the experimental protocol was approved by the local ethics committee. All subjects gave their written informed consent for the study.

\section{STIMULUS MATERIAL}

The cerebral organization of language at the word and sentence level has been investigated extensively with PET, fMRI, MEG, and event-related potential studies, but much less research has to date been carried out on the processing of coherent language at the discourse level where language occurs in its natural context (i.e., where phonetic, syntactic, semantic, and pragmatic aspects of language are integrated). Therefore, in this study we adopted coherent spoken speech (radio news) as stimuli and used them in a listening comprehension and discourse processing paradigm. In cooperation with the English department at the University of Vienna, the speech samples were matched for syntactic complexity, semantic content, and genre (only reports of medium complexity level on daily politics and business were chosen), discourse structure (reports had the form of a monolog), and gender of the speaker (all male speakers). Within the framework of a block design, six blocks of coherent speech (2.0-3.2 min each) with randomly inserted baseline blocks (acoustic noise, $2.0 \mathrm{~min}$ each) were presented in randomized order: three blocks in condition L2 English and three blocks in condition L1 German were auditorily presented in randomized order. All stimuli were presented via earphones and a white fixation cross was presented throughout the auditory task. For visualization of stimulus presentation procedure see Figure 1.

The whole recording session, which began at the same time of day for each participant (9 O'clock a.m.), took approximately $3 \mathrm{~h}$ for each participant, including manual electrode placement, explanation of the procedure, personal questionnaires, a familiarization task, and interruptions for answering detailed open ended comprehension questions orally performed with an experimenter (a tutor of the English Department) who was blind regarding group membership. In these interview sessions, six psychological reaction parameters were assessed with the help of a behavioral questionnaire, comprising (1) The actual text comprehension (seven factual comprehension questions about the contents of the radio report were posed, 14 points $=\max$ score, $0=$ min score), (2) Subjective text comprehension (participant scored himself on a rating scale from 1 to 5), (3) Self-reported attention (same procedure), (4) Cognitive work-load (same procedure), (5) Sympathy for the speaker (same procedure), and (6) Interest in the subject matter of the radio fragment (same procedure).

\section{DATA RECORDING}

We recorded multivariate EEG signals during L1 and L2 processing in a quiet, dimly lit sound-proof experimental room. Participants were monitored through a video control system during the recording session in order to control for possible movements. Nineteen gold-disk electrodes were carefully attached to the scalp with adhesive electrode gel, positioned according to the international 10/20 System (Jasper, 1958; Figure 2); one additional frontal electrode was used as a ground, and two separate electrodes, at the right and left ear-lobe, were used as reference electrodes. The recordings were referenced against the algebraic mean of the two ear-lobe electrodes (Essl and Rappelsberger, 1998). Eye movements were additionally controlled for by a piezo-electric device attached to the eyelid. Using a conventional Nihon-Kohden 21 channel recorder, the EEG was amplified, filtered (time constant $0.3 \mathrm{~s}$ ),

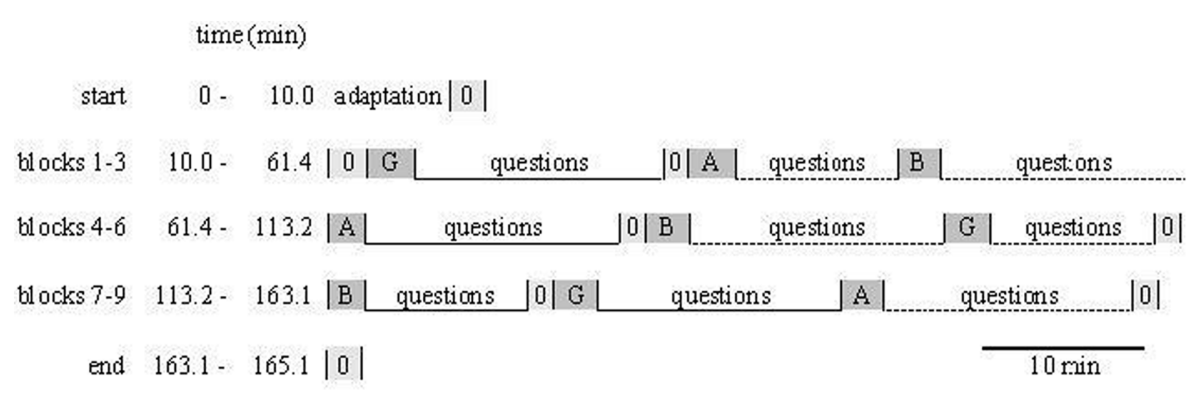

FIGURE 1 | Timing of recording session (example). Three tasks of the different language varieties $(B=$ British English, $A=$ American English, or $\mathrm{G}=$ Austrian German) were linked together to one big block comprising three small blocks with their respective baseline conditions, and the three big blocks $(1-3,4-6,7-9)$ were presented in randomized order. After each language task (acoustic presentation), recording was interrupted for questions to explore factual listening comprehension, the attitude toward the speaker, interest for the contents, attention, work-load, and subjective comprehension $(0=$ baseline task). 


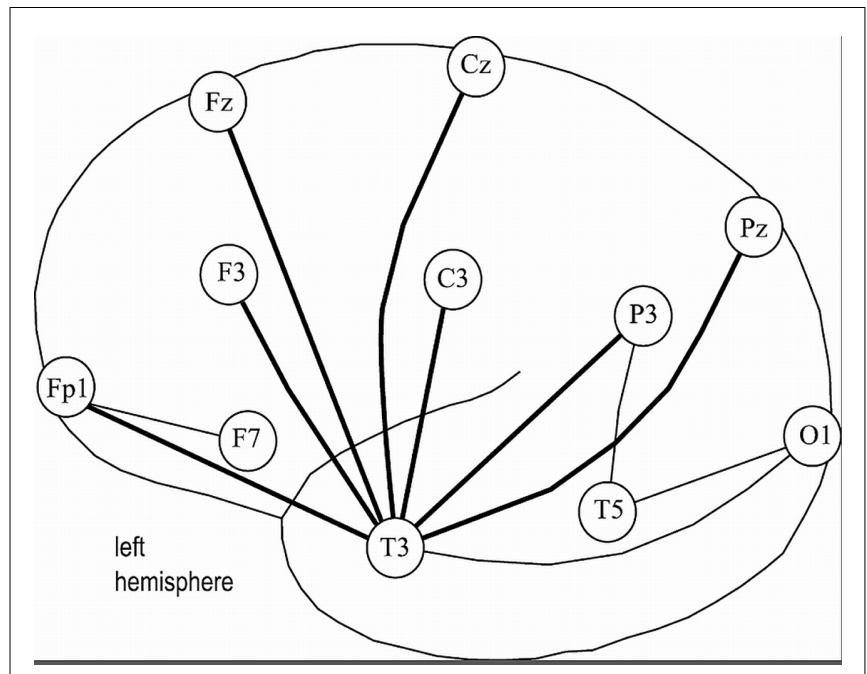

FIGURE 2 | Schematic map of the left hemisphere of EEG electrode

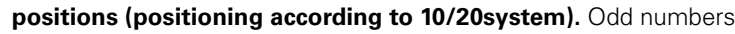
represent loci in the left hemisphere, even numbers loci in the $\mathrm{RH}$ respectively. $\mathrm{Fp}=$ fronto-polar region, $\mathrm{F}=$ frontal lobe, $\mathrm{C}=$ central region, $\mathrm{P}=$ parietal lobe, $\mathrm{T}=$ temporal lobe, $\mathrm{O}=$ occipital lobe. Index letter " $\mathrm{z}$ " means "zero" for midline (central line, vertex). Indexing numbers on the $\mathrm{RH}$ would be: $4,8,2$, and 6 instead of 3, 7, 1, and 5 respectively.

displayed and recorded at a sampling rate of $128 \mathrm{~Hz}$ for further processing. The electrode impedance was kept below $5 \mathrm{k} \Omega$. A notch at $50 \mathrm{~Hz}$ was used for the elimination of power line contamination. We applied two criteria for possible artifact rejection: we rejected those epochs with amplitudes higher than $70 \mu \mathrm{V}$ (absolute value), plus additional epochs where $2 \%$ or more samples deviated more than $3 \mathrm{SD}$ from the mean value.

\section{DATA ANALYSIS}

Phase synchronization (PS) between all possible electrode pairs $[(19 \times 18) / 2=171$ different electrode pairs $]$ was calculated in the lower gamma frequency range $(30-45 \mathrm{~Hz}$, the choice was made after earlier studies, see also Bhattacharya et al., 2001; Bhattacharya et al., 2003; Bhattacharya and Petsche, 2005a,b) by three methods: (i) global PS by coarse-graining of Markov chains (CGMC) which measures the degree of PS of one electrode with all other electrodes, (ii) bivariate PS [by the recently developed phase lag index (PLI)] which measures the degree of PS between pairs of electrodes, and additionally (iii) the more conventional mean phase coherence, which measures the degree of PS between a pair of electrodes, but is more prone to volume conduction effects. However, all three methods initially require a proper estimation of the phases from the EEG signals.

\section{Estimation of the phases}

Since we were mainly interested in the gamma frequency band, each EEG signal was band-pass filtered using a zero-phase filter with 30 and $45 \mathrm{~Hz}$ cut-off frequencies to get the desired gamma band signal. We calculated the phases of these filtered signals, $\{x(k)\}$, by using the analytic signal approach based on Hilbert transform $(\mathrm{HT})$, where the analytical signal $\zeta(t)$ is obtained:

$\zeta(t)=x(t)+i x_{\mathrm{H}}(t)$

where $x_{\mathrm{H}}(t)$ is the HT of $x(t)$, defined as:

$x_{H}(t)=\frac{1}{\pi} P \cdot V \cdot \int_{-\infty}^{\infty} \frac{x(t)}{t-t^{\prime}} d t^{\prime}$

with P.V. denoting the Cauchy principal value.

The analytic signal, which is also a complex function, can be decomposed as:

$\zeta(t)=a_{x}(t) \exp ^{i \phi_{x}(t)}$

where $a_{x}(t)$ is the instantaneous amplitude and $\phi_{x}(t)$ is the instantaneous phase of $x(t)$.

In this way, the phases of 19 EEG channels, $\phi_{i}(t)(i=1, \ldots, 19)$, were estimated and subsequently used to assess the degree of PS in each situation, as explained below.

\section{Estimating global phase synchronization: Coarse-Graining of Markov Chains (CGMC)}

The collective synchronization of the ensemble of 19 electrodes was studied by means of a recently derived method (CGMC; Allefeld, 2006; Allefeld and Bialonski, 2007). CGMC is a multivariate method that allows the detection of synchronization clusters from the $19 \times 19$ matrix of bivariate PS indexes (in our case, PLI). Briefly, this matrix is translated into a stochastic matrix $P$ describing a finite-state Markov process, and subsequently, it is possible to estimate the number of clusters present in the data via the eigenvalue decomposition of $P$. Additionally, it allows the estimation of the strength of each cluster as well as the degree of participation of each electrode in the cluster it belongs to.

The relevant fact about CGMC is that it is truly multivariate in the sense that, given a set of $n$ electrodes $(n>2)$, it estimates the degree of overall synchronization among all the electrodes and their distribution in $q$ synchronization clusters $(q \geq 1)$ to which each electrode of contributes differently. The validity of this approach in EEG applications has been demonstrated (Allefeld and Kurths, 2004; Allefeld and Bialonski, 2007).

Although the CGMC allows an automatic determination of the value of $q$ from the data, after a preliminary exploration we fixed $q=2$ so that the 19 electrodes are assigned to either the strongly synchronized or the weakly synchronized cluster.

\section{Estimating bivariate phase synchronization: phase lag index (PLI)}

There are many different ways of assessing the PS between a pair of EEG signals (see, e.g., (Pereda et al., 2005)). Here, we used the PLI (Stam et al., 2007), because it is less sensitive to volume conduction effects than other popular indexes of PS such as, for instance, the mean phase coherence (Mormann et al., 2000). The PLI is defined as:

$\mathrm{PLI}=\mid\left\langle\operatorname{sgn}\left(\varphi\left(t_{k}\right)\right\rangle\right|$

where $|\bullet|$ indicates modulus, $\langle\bullet\rangle$ indicates time average and

$\varphi(t)=\left|\phi_{i}(t)-\phi_{j}(t)\right| \bmod (2 \pi)$ 
is the cyclic relative phase, i.e., the phase difference between $x_{i}(t)$ and $x_{j}(t)$ wrapped to the interval $[0,2 \pi]$. The PLI ranges from 0 (two signals with no phase relationship or a phase relationship symmetrical about 0 or $\pm \pi$-which is a signature of volume conduction effects (Nolte et al., 2004; Stam et al., 2007) to 1 (two signals with complete phase synchrony); PLI is parameter free.

\section{Estimating bivariate phase synchronization (additional): mean phase coherence}

Additionally, we also used the now more conventional mean phase coherence (Hoke et al., 1989; Mormann et al., 2000) defined as:

$\gamma_{i, j}=\sqrt{\langle\cos \varphi(t)\rangle^{2}+\langle\sin \varphi(t)\rangle^{2}}$

where $\langle\bullet\rangle$ indicates time average and

$\varphi(t)=\left|\phi_{i}(t)-\phi_{j}(t)\right| \bmod (2 \pi)$

is the cyclic relative phase, i.e., the phase difference between $x_{i}(t)$ and $x_{j}(t)$ wrapped to the interval $[0,2 \pi]$. The mean phase coherence index ranges from 0 (two signals with no phase relationship) to 1 (two signals with complete phase synchrony), and has the advantage of being parameter-free.

\section{STATISTICAL ANALYSIS}

The statistical differences in the synchronization strength of the strongly and the weakly synchronized cluster was tested by means of repeated measures ANOVA test with proficiency (HP and LP) as independent (between groups) factor and language processing (L1 and L2) as dependent (within group) factors. Differences were considered significant when the $p$-value was lower than 0.05 .

For the sake of using balanced stimulus trials in the group comparisons of L1 versus L2, we only analyzed the three blocks of the condition "British English" versus the three blocks of "Austrian German," based on our earlier experience (Reiterer et al., 2005a) that the variant of English (British or American English) neither affected the coherence patterns in the EEG nor the respective behavioral outcomes. Thus it had emerged previously (as far as it can be discriminated by EEG synchronization analyses) that L2 English was processed as L2 English and not differentiated further into its subvariants or accents.

\section{RESULTS}

\section{BEHAVIORAL RESULTS}

A comprehension questionnaire applied after each task condition revealed (Figure 3) that the low proficiency (LP) group understood approximately $50 \%$ of the English (L2) texts, whereas the HP group understood nearly perfectly (95\%). For the comprehension questions of the control condition German (L1) no statistical difference between the groups was obtained. The HP group had again a performance accuracy of 95\%, whereas the LP group scored slightly, but not significantly, worse (performance accuracy of $80 \%$ ).

No differences between the two groups were found for other psychometric variables (self-reported attention, work-load, sympathy for the speaker, and interest in the subject matter).

\section{EEG SYNCHRONIZATION RESULTS}

First we could differentiate L1 from L2 processing by gamma band global synchronization clustering patterns (CGMC) but on a more subtle scale, HP and LP bilinguals could further be significantly differentiated by bivariate gamma band synchronization measures (gamma band mean phase coherence and PLI) predominantly, or, almost exclusively when processing L2.

On a global scale, i.e., when estimating the "global PS" by the method of CGMC where the collective synchronization of the ensemble of 19 electrodes is studied with respect to cluster strength, we obtained very similar general results of clustering strength for both groups investigated (high and low proficiency group alike), however significantly different for the two languages, mother tongue German, and second language English. Cluster strength is significantly higher in both groups for the L2 than for the L1 (Figure 3).

When taking a more fine-grained view for the distribution of the electrodes belonging to the stronger cluster within the gamma band (measured with bivariate PS by means of the "PLI" which measures the PS between the single pairs of electrodes), we obtained more subtle differences in synchronization characteristics between the high and the low proficiency group.

The topography is markedly different between the HP and the LP group (Figure 4) with the LP group showing a strongly and significantly synchronized cluster only for processing L2 within left temporo-parietal areas preponderantly. The other topographical clusterings (areas) shaded in light blue over central and right hemisphere areas did not reach significance. To work out these between group differences in detail, we provided an additional figure (Figure 5) where these group differences in PLI are depicted in percentages (percentages of pertinence to the cluster) - the positive values (yellow/red color) corresponding to those electrodes

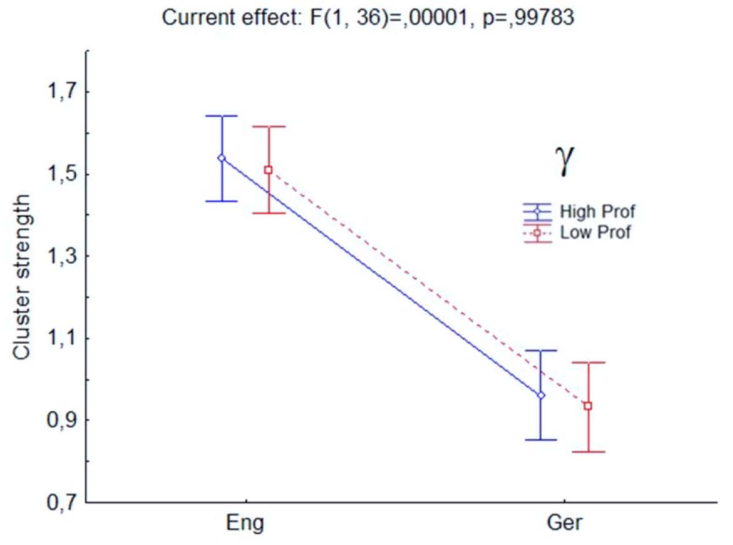

FIGURE 3 | Synchronization cluster (strength) analysis by "Coarse-Graining Markov Chains" (CGMC) within the $(\gamma)$ gamma-band $(30-45 \mathrm{~Hz})$ showed significant differences between native language German (Ger, $x$-axis), cluster strength ( $y$-axis) around 0.9 , and second language English (Eng) with cluster strength significantly higher (around 1.5). Red line denotes the low proficiency and blue line the high proficiency group. 
which belong to the strong cluster in a greater percentage to subjects in the LP group. In the LP group one can see a marked increase in the left temporo-parietal/central region while listening to L2 English whereas the increase is much lower and right temporal for the listening of German (Figure 5).

According to this result for the L1 we can assume that an increase up to $20 \%$ is within the statistical fluctuation (no difference is expected usually between the groups while listening to L1), so that an increase of $25 \%$ or above might be considered significant. These results indicate that the low proficiency group recruits more often the left temporo-parieto-central part of the cortex than the HP group when listening to L2 English, which is clearly also their less proficient language.

To obtain a topographical scalp distribution of the group differences in average participation of electrodes for the strongly synchronized cluster (PLI, within the gamma band) we calculated an index for the participation of each electrode and subtracted the values of the HP group from those of the LP group (Figure 6). Here we found that the greatest increment of involvement of the electrodes belongs to the LP group during the L2 language condition and is topographically most pronounced over left frontal, or fronto-central areas.

The positive values (red color) here indicate a higher participation of electrodes for the low proficiency group, again with a preponderance over the left hemisphere and stronger for L2 than for L1. The topographies are significantly different for the groups $(p<0.01$, sign ranked paired test) when listening to the second language English, whereas for listening to the native language topographies are not significantly different between the groups (average difference is $5 \times 10^{-4}$, i.e., equal to zero).

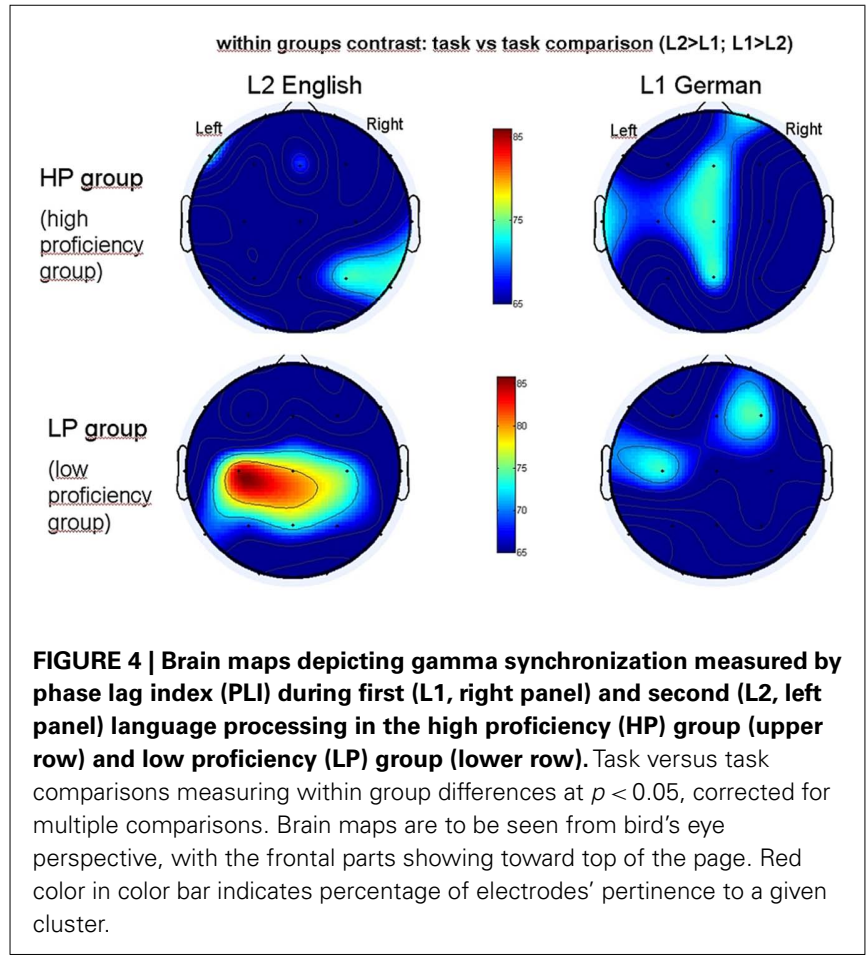

To get an impression about the most importantly involved electrodes, we depicted the results described in Figure $\mathbf{6}$ additionally in a topographical map showing synchronization lines in the LP group (connections are given for interhemispheric longrange connections and within left/right hemisphere separately). Within the strongest participating left hemispheric frontal cluster, the greatest involvement of long-range interhemispheric gamma synchronization concerns the electrode positions: F3, F7, Fp1 to $\mathrm{C} 4, \mathrm{P} 4$, and $\mathrm{T} 3$ to $\mathrm{F} 4$. Within the right hemisphere only (corresponding to the right panel in Figure 6) the long-range connections involve electrode positions connecting frontal with parietal regions.

The results for the gamma band synchronization differences between the high and low proficiency group were corroborated by an additional analysis (Figure 8, calculation of mean phase coherence, see Estimating Bivariate Phase Synchronization (Additional): Mean Phase Coherence in Materials and Methods).

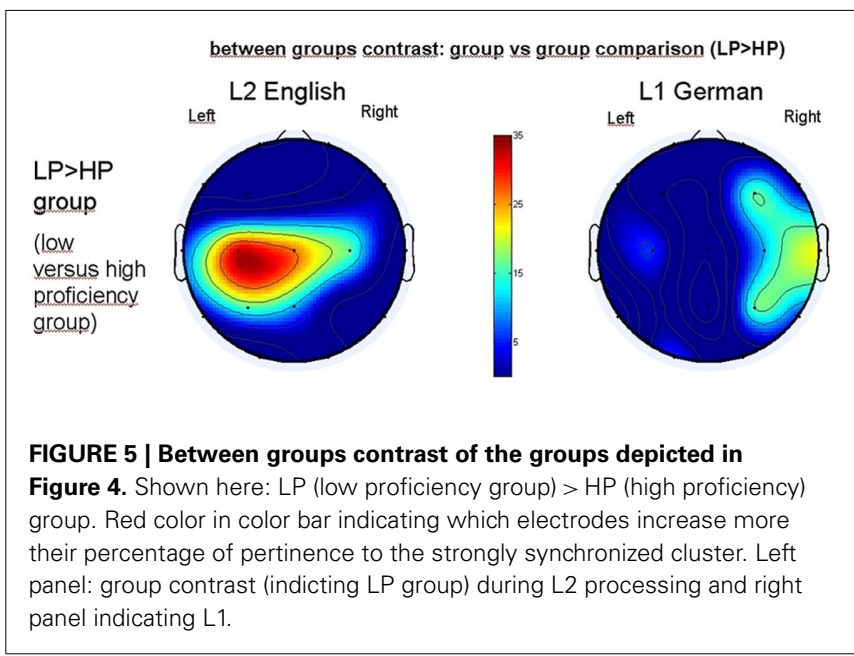

average participation index of each electrode in the stronger cluster
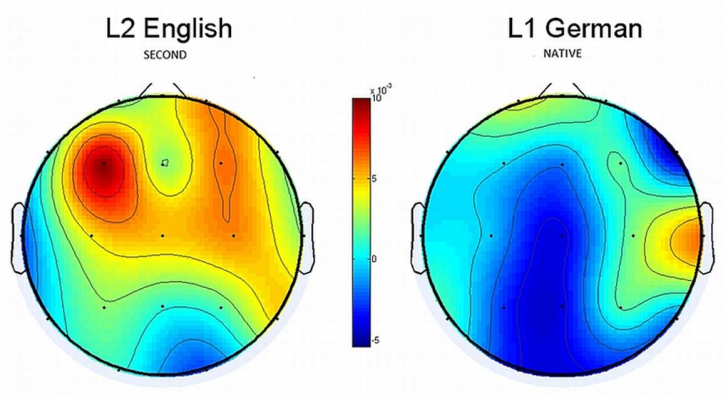

between groups contrast: group vs group comparison (LP>HP)

FIGURE 6 | Topography distribution brain maps of the average participation of each electrode (synchronization cluster index) in the strongly synchronized cluster within the gamma band detected by PLI. Comparison shown here: LP > HP group (group contrast, HP subtracted from LP group). Color bar (red color) indicating the greatest increment of involvement of the electrodes in left frontal/fronto-central areas during L2 (left panel) processing in the LP group. 


\section{gamma synchronization patterns for low proficiency (LP>HP) groug:}
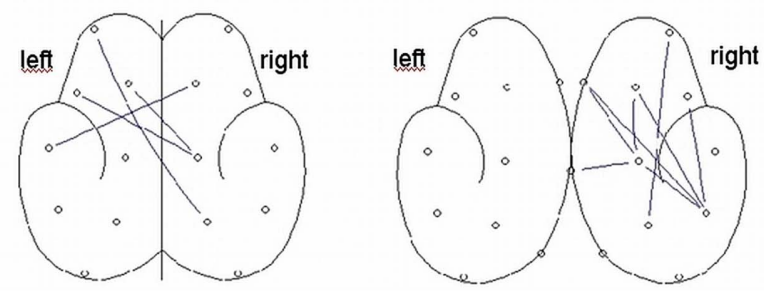

FIGURE 7 | Connectivity brain maps visualizing the most synchronized and exact electrode positions (synchronization patterns) within the strongly synchronized cluster in the gamma band for the interhemispheric connections only (left panel) and the within hemisphere (left and right) connections, for the condition L2 English in the LP > HP group.

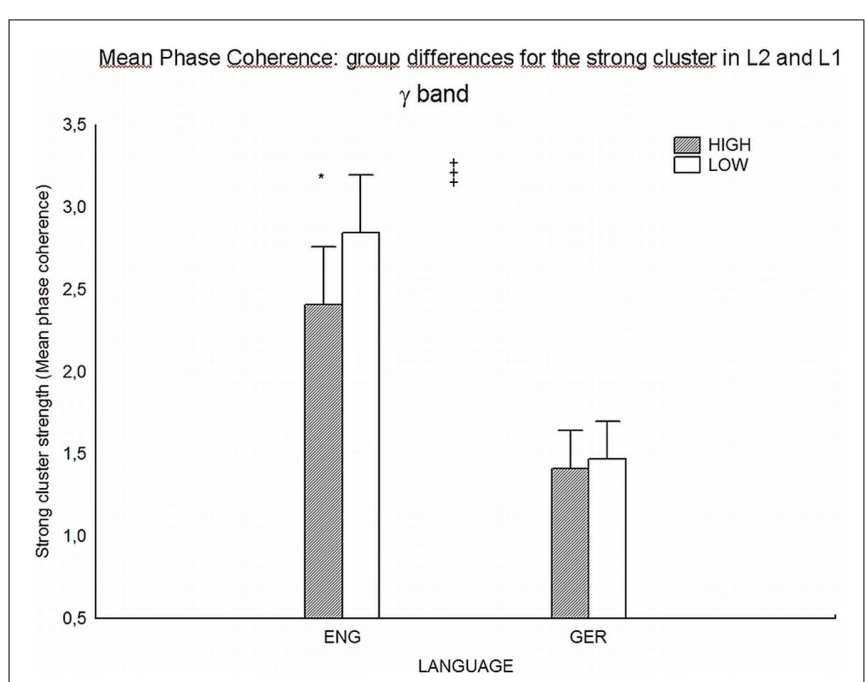

FIGURE 8 | Mean Phase Coherence: differences between the groups during L2 ("ENG," left double bar) and L1 ("GER," right double bar) processing. Dark bars indicate the high proficiency group and white bars the low proficiency group. Difference in mean phase coherence is significant ( $p=0.42$ ) between the groups during processing L2 English (increased synchronization strength for LP group), but not during processing L1 German

Where the mean phase coherence yields a significant difference between the HP and LP group ( $p=0.42$ ) for the strongest synchronized gamma-band cluster during the processing of English L2, the same significant group difference cannot be found for native language German. This analysis corroborates the main finding for stronger synchronization in gamma band during processing of L2 for the lower proficiency group.

\section{DISCUSSION}

In this study, we primarily showed that different levels of "cortical control" or cortical processing mechanisms accompany the processing of second and first language, and furthermore, that differently proficient bilinguals can be differentiated by their cortical connectivity patterns, especially while processing their less fluent language. We suppose that ease of language processing might in part be instantiated by the brain trough different levels of synchronization between language network areas, with stronger synchronization between larger and more extended networks reflecting the recruitment of more resources (cortical effort), either because the task is generally more difficult as in the case of processing a later learned second language by late bilinguals or - on a more subtle scale - because proficiency differences due to differences in long-term language training make the task at hand more effortful and hence call for the integration of more global workspace in the brain.

Generally speaking, our results offer two insights (one more of a factual, one more of a methodological nature): first, language learners who are highly proficient in their L2 and have undergone extensive linguistic training seem to use different language processing strategies reflected in different cortical patterns on the level of synchronized electrophysiological activity in the brain, and secondly, these behavioral differences in cognitive processing can be made visible by measuring synchronized activity within the EEG gamma frequency range. Our results indicate a speculative role of gamma band as a further method to investigate the neural substrate of bilingual proficiency level.

\section{EEG COHERENCE/SYNCHRONIZATION PATTERNS IN LATE BILINGUALS}

By employing a recently derived technique of EEG global synchronization analysis (CGMC, see Data Analysis) we found pronounced differences in synchronization strength in the gamma frequency range between auditory text processing/comprehension in mother tongue and a later learned (around 9 years of age) second language. This result points to subtle differences in cortical control mechanisms at the level of interconnectedness between language areas and surrounding tissue and their connectivity patterns - possibly as a function of differences in "language entrenchment" (Mac Whinney, 2010).

Cooperative activity, interactions and communication between neuronal assemblies through coherent oscillations subserving cognitive processes have been traced and investigated with electrophysiological methods by means of coherence and synchronization analyses within various frequency ranges of the ongoing brain responses (Engel and Singer, 2001; Ward, 2003; Fries, 2005). Increased synchronization between and within frequency ranges (e.g., most prominently alpha, theta) was found to reflect increased working memory demands, short-term memory work-load, and cognitive effort (Sarnthein et al., 1998; Sauseng et al., 2005; Schack et al., 2005). Recently gamma band analyses of the human EEG have become very promising sources for gaining new insights into higher-order cognitive information processing. What formerly has been discarded, or cut-off as "noise" or contaminated EEG, is now looked upon as a valuable tool for investigating the most sophisticated mental processes including music perception (Bhattacharya and Petsche, 2005b) and artistic imagination (Bhattacharya and Petsche, 2005a). Gamma band oscillations (an indicator of local or short-range synchronization) are said to reflect gestalt perception (Kaiser and Lutzenberger, 2003) or a kind of matching process between bottom-up and top-down information (e.g., comparing memory contents with incoming stimulus related information 
(Herrmann et al., 2004). Further, gamma band PS (an indicator of long-range synchronization) is thought to reflect cognitive "binding" phenomena, feature integration, STM, higher-order integrated thinking associated with quick high-density information processing, and transient associations of neural assemblies (Fell et al., 2003).

Within the domain of language, some authors have already investigated the role of gamma band oscillations in (native) language processing (Pulvermüller et al., 1997), for example, for the syntactic and semantic domain (Braeutigam et al., 2001; Micheloyannis et al., 2003; Hagoort et al., 2004; Ihara and Kakigi, 2006; Bastiaansen et al., 2010) for verbal performance and intelligence (Jausovec and Jausovec, 2005) as well as for correlations with semantic complexity (Simos et al., 2002). In addition, gamma band oscillations and synchronization phenomena have also been reported for L1 processing (Ford et al., 2005; Weiss et al., 2005; Ihara and Kakigi, 2006). For example, Ford et al. (2005) found that binding mechanisms in sentence processing were reflected in fronto-temporal gamma synchrony. Effects of sentence complexity on gamma coherence have been reported by the Weiss et al. (2005) study. Although all these EEG coherence studies were performed purely on native language processing, it seems not too far-fetched to compare L1 with L2 phenomenologically and look for bilingual language processing as well in the gamma frequency range. We would like to argue that it is only a logical consequence that bilingual language processing and cortical control mechanisms that are related to individual differences in the mastery of languages, can be revealed by adopting the above described method. We suggest that the basic mechanisms which underlie second language processing are similar to the ones in first language processing from a theoretical and empirical point of view, e.g., (Newman-Norlund et al., 2006), since it is compatible with recent brain imaging studies which find (at least partially) overlapping areas of activation for L1 and L2 (Hasegawa et al., 2002; Chee et al., 2003; Marian et al., 2003; Vingerhoets et al., 2003; Lucas et al., 2004; Ojima et al., 2005; Reiterer et al., 2005a,b, 2009; Klein et al., 2006; Gandour et al., 2007). Bilingual brain organization in terms of networks and functional connectivity has rarely been investigated so far (for EEG coherence see Reiterer et al., 2005a,b). With the method of fMRI connectivity the only studies to date are a study by Dodel et al. (2005) and Majerus et al. (2008). Both of them investigated cortical synchronization patterns by employing fMRI connectivity analyses in bilingual language processing (Dodel et al., 2005) and native language STM processing (Majerus et al., 2008). Interestingly, the Dodel et al. found bigger and more extended networks for the bilinguals with higher proficiency levels with fMRI connectivity, contrary to many studies on bilingual fluency levels, which find fewer activated areas as a function of higher fluency levels in second languages (e.g., Perani et al., 1996, 1998; Yetkin et al., 1996; Chee et al., 2001; Hasegawa et al., 2002; Briellmann et al., 2004; Xue et al., 2004; Klein et al., 2006). The very recent study by Majerus et al. (2008) could however differentiate high from low proficiency bilinguals by fMRI connectivity patterns.

\section{DIFFERENCES IN PROFICIENCY LEVEL}

However, we did not only find significant differences in connectivity strength between first and second language, but also between the different proficiency groups. This finding per se appears to be rather intuitive, since the participants in our study were no early bilinguals, but mixed proficiency late bilinguals who were exposed to the second language for the first time around 9 years of age. More importantly than age of onset even (Birdsong, 2006), they received most of their foreign language input through formal classroom training and very little through natural exposure in an L2 setting. Hence, we believe that the reason for this striking difference in gamma band synchronization strength (Figure 3 ) is mostly due to differences in exposure, entrenchment, and language learning methods, less to age of onset of learning the language. The recent brain imaging literature on bilingual or multilingual language learning increasingly supports the viewpoint that proficiency differences have more impact on brain organization in bilinguals than "pure" age of onset (Kotz, 2009; Reiterer, 2010). Proficiency on the other hand is a "fuzzy" term insofar as it needs to be clarified in the first place which factors led to a certain level of proficiency (be it a special long-term exposure, an early onset, an intensive training paradigm, a special aptitude or predisposition for language learning etc.). Differences in proficiency level can be reached by various different factors, or, more realistically, a combination of those.

By employing further analyses in the gamma range on the two groups we investigated (proficiency levels due to different amounts and quality of language training) we found differences in connectivity patterns reflecting the differences in level of fluency/proficiency in L2.

Differently proficient bilinguals, who had either higher or lower amounts of linguistic training and expertise in their second language, could be differentiated by their EEG network activity or synchronization patterns in the gamma frequency band by mean phase coherence analysis and topographical differences of the underlying employed networks by means of PLI. More specifically, we found that that during processing the second language, the low proficiency bilinguals, as compared to HP bilinguals, produced more strongly synchronized patterns of functional connectivity especially in left fronto-parietal areas. The low proficiency speakers seemed to recruit those areas in a concerted manner more often than their HP counterparts.

Our findings related to proficiency differences in L1 and L2 processing, are based on two results: (1) group $\times$ language analysis (Figure 3) mainly reflecting differences of the two language systems (L1 and L2) on a cortical processing level (increased synchronization strength in L2 for both groups), and (2) within and between groups analysis (Figures 4-7) indicating that linguistic training can alter L2 processing demands on a cortical as well as on a behavioral level and less proficient second language users have to recruit broader language networks (in left fronto-parietal areas) more strongly (with higher connection strength).

\section{BILINGUAL LANGUAGE PROCESSING AND THEORIES OF CORTICAL EFFICIENCY}

What has been observed many times in various domains outside of language processing that increased cognitive demands are accompanied by increased activity levels or extended area recruitment (e.g., for intelligence see Haier et al., 1988; Haier et al., 1992; Grabner et al., 2006, for music processing: Lotze et al., 2003, for working 
memory: Sarnthein et al., 1998; Sauseng et al., 2005), has also been reported for L1 as well as L2 processing (Raichle et al., 1994; Just et al., 1996; Yetkin et al., 1996; Perani et al., 1998; Rypma and D'Esposito, 1999; Dräger et al., 2004; Xue et al., 2004; Reiterer et al., 2005a,b; Abutalebi, 2008; Kotz, 2009; Leonard et al., 2011). What has been explicitly called "cortical efficiency" could be termed "proficiency level differences" in the field of L2 processing. With native language processing several authors found that comparable to a "compensation mechanism," brain activation increases with the complexity in linguistic processing (Just et al., 1996; Rypma and D'Esposito, 1999; Dräger et al., 2004) or reduces with increased repetition and practice (Raichle et al., 1994; Thompson-Schill et al., 1999). In the field of second language processing as well, various research groups have detected the "cortical efficiency" phenomena by revealing that proficiency level (either attained by practice, higher exposure or by formal training or as occurring more naturally by both) has an influence on the extent and intensity of cortical activation in a bilingual's brain (Yetkin et al., 1996; Perani et al., 1998; Chee et al., 2001; Hasegawa et al., 2002; Wartenburger et al., 2003; Briellmann et al., 2004; Xue et al., 2004; Zhang et al., 2005; Klein et al., 2006). The usual observation can be summarized in the following terms: lower proficiency, more distributed activity (i.e., a Larger network) and higher proficiency, more focal activity (smaller network). Our results of higher gamma band long-range synchronization in L2 going hand in hand with lower proficiency level in bilinguals and lower gamma synchronization with higher proficiency level, are pointing into the same direction, possibly revealing a compensation mechanism in the domain of second language processing.

\section{LIMITATIONS OF THE STUDY}

The current study also has a few limitations. No verbal or nonverbal IQ test was performed as control, because of limitations of time and laboratory use and the theoretical consideration that language abilities do not correlate with non-verbal intelligence. We want to point to this shortcoming and are aware that this might limit the interpretation of our results. It the same vein it needs to be mentioned that we carefully chose two groups with a closely matching educational level, preselected by university exams (participants were all students with completed Bachelor's degree, studying for a Master's) and this might enhance group similarity with respect to higher cognitive and intellectual abilities.

Finally, we would like to mention one additional point in here, which could be regarded as limitation or as interesting outcome likewise. This is the behavioral result that our two groups behaved slightly differently already when tested (comprehension questions) in their mother tongue. The low proficiency group with regard to L2 scored slightly worse when tested on L1 comprehension and text recall. This we like to call "the L1 paradox." Usually the L1 is implemented as a control condition where the groups should behave in exactly the same way, because native speakers are perceived as a "homogeneous mass." This is the classical intuition, but our data as well as other research (Pakulak and Neville, 2010; Reiterer et al., 2011) show that also mother tongue speakers can differ in their L1 proficiency and competence levels (a fact which is also affirmed by the existence of congenital language disorders). Variation within L1 competence might be smaller than within L2 interlanguages, but nevertheless existing. Recent research shows that also smaller differences in L1 competence and ability levels can be traced by brain imaging techniques and detected in such a way which would not have been possible with pure behavioral measurements. Even in the case of our present study, the result of the L1 differences behaviorally was just a marginal one, statistically speaking only a "trend." Such small differences in mother tongue processing can, however, point to important underlying principles. What they show is individual differences in the linguistic abilities of L1 speakers. One of the theoretical concepts that tries to capture this phenomenon is language aptitude. Theoretical assumptions and new brain research data likewise (Wells, 1985; Abrahamsson and Hyltenstam, 2008; Golestani et al., 2011; Reiterer et al., 2011) show that the variable of general language aptitude is a possible hidden driving force behind individual differences in L1 as well as L2 proficiency and ability levels. Very often, this variable is neglected in the whole field of bilingualism research, be it behavioral or neurocognitive. What we could have hit upon in our present study by detecting those small L1 trend differences, is pre-existing differences between the groups in language aptitude. General language aptitude might also drive and determine career and study choice. In our case here aptitude might have partly driven the language students to study foreign languages and linguistics (our HP group). We acknowledge that it is very difficult to "control" for all these pre-existing variables, but at the same time it needs to be said that they might be very important in explaining a lot of variance in bilingual data. Thus, what is missing in many studies of bilinguals or/and second language learners (including the present study) is a sound testing for individual differences in language aptitude. This is a methodological as well as a theoretical issue and an important point to be considered in future studies.

\section{CONCLUSION}

We have shown here that by looking at EEG gamma band phase synchronization patterns, one can differentiate second (classroomlearned) from first language, and within the later learned second language (L2) learners with lower amounts of linguistic training and expertise from those with higher amounts of expertise by the different ways by which they employ synchronized activation. The observed patterns could be explained by the theory of cortical efficiency because we found different network patterns for high and low proficiency learners, with more widely distributed synchronized networks in left fronto-parietal areas more often recruited by lower proficiency learners. Our findings further indicate that EEG gamma band phase synchronization measures are sensitive to differences in second language processing and control strategies due to experience/proficiency-driven differences.

\section{ACKNOWLEDGMENTS}

Joydeep Bhattacharya is supported by JST.ERATO project. The author Susanne Reiterer is supported by the German Research Foundation (DFG, project AC-55/7-1). 


\section{REFERENCES}

Abrahamsson, N., and Hyltenstam, K. (2008). The robustness of aptitude effects in near-native second language acquisition. Stud. Sec. Lang. Acquis. 30, 481-509.

Abutalebi, J. (2008). Neural aspects of second language representation and language control. Acta Psychol. (Amst) 128, 466-478.

Allefeld, C. (2006). About the derivation of the SCA algorithm. Int. J. Bifurcat. Chaos 16, 3705-3706.

Allefeld, C., and Bialonski, S. (2007). Detecting synchronization clusters in multivariate time series via coarse-graining of Markov chains. Phys. Rev. E 76, 066207.

Allefeld, C., Frisch, S., and Schlesewsky, M. (2005). Detection of early cognitive processing by eventrelated phase synchronization analysis. Neuroreport 16, 13-16.

Allefeld, C., and Kurths, J. (2004). An approach to multivariate phase synchronization analysis and its application to event-related potentials: synchronization cluster analysis. Int. J. Bifurcat. Chaos 14, 417-426.

Bastiaansen, M., and Hagoort, P. (2006). Oscillatory neuronal dynamics during language comprehension. Prog. Brain Res. 159, 179-196.

Bastiaansen, M., Magyari, L., and Hagoort, P. (2010). Syntactic unification operations are reflected in oscillatory dynamics during online sentence comprehension. J. Cogn. Neurosci. 22, 1333-1247.

Bhattacharya, J., Pereda, E., and Petsche, H. (2003). Effective detection of coupling in short and noisy bivariate data. IEEE Trans. Syst. Man Cybern. B 33, 85-95.

Bhattacharya, J., and Petsche, H. (2005a). Drawing on mind's canvas: differences in cortical integration patterns between artists and non-artists. Hum. Brain Mapp. 26, 1-14.

Bhattacharya, J., and Petsche, H. (2005b). Phase synchrony analysis of EEG during music perception reveals changes in functional connectivity due to musical expertise. Signal Process. 85, 2161-2177.

Bhattacharya, J., Petsche, H., and Pereda, E. (2001). Long-range synchrony in the gamma band: role in music perception. J. Neurosci. 21, 6329-6337.

Birdsong, D. (2006). Age and second language acquisition and processing: a selective overview. Lang. Learn. 56, 9-49.

Braeutigam, S., Bailey, A. J., and Swithenby, S. J. (2001). Phase-locked gamma band responses to semantic violation stimuli. Brain Res. Cogn. Brain Res. 10, 365-377.
Briellmann, R. S., Saling, M. M., Connell, A. B., Waites, A. B., Abbott, D. F., and Jackson, G. D. (2004). A high-field functional MRI study of quadri-lingual participants. Brain Lang. 89, 531-542.

Chee, M. W., Hon, N., Lee, H. L., and Soon, C. S. (2001). Relative language proficiency modulates BOLD signal change when bilinguals perform semantic judgments. Blood oxygen level dependent. Neuroimage 13, 1155-1163.

Chee, M. W., Soon, C. S., and Lee, H. L. (2003). Common and segregated neuronal networks for different languages revealed using functional magnetic resonance adaptation. J. Cogn. Neurosci. 15, 85-97.

De Bot, K. (2008). The imaging of what in the multilingual mind? Review article. Sec. Lang. Res. 24, 111-133.

Dodel, S., Golestani, N., Pallier, C., Elkouby, V., Le Bihan, D., and Poline, J. B. (2005). Condition-dependent functional connectivity: syntax networks in bilinguals. Philos. Trans. $R$ Soc. Lond. B Biol. Sci. 360, 921-935.

Dräger, B., Jansen, A., Bruchmann, S., Forster, A. F., Pleger, B., Zwitserlood, P., and Knecht, S. (2004). How does the brain accommodate to increased task difficulty in word finding? A functional MRI study. Neuroimage $23,1152-1160$.

Engel, A., and Singer, W. (2001). Temporal binding and the neural correlates of sensory awareness. Trends Cogn. Sci. (Regul. Ed.) 5, 16-25.

Essl, M., and Rappelsberger, P. (1998). EEG Coherence and reference signals: experimental results and mathematical explanations. Med. Biol. Eng. Comput. 36, 399-406.

Fell, J., Fernandez, G., Klaver, P., Elger, C. E., and Fries, P. (2003). Is synchronized neuronal gamma activity relevant for selective attention? Brain Res. Brain Res. Rev. 42, 265-272.

Ford, J. M., Gray, M., Faustman, W. O., Heinks, T. H., and Mathalon, D. H. (2005). Reduced gamma-band coherence to distorted feedback during speech when what you say is not what you hear. Int. J. Psychophysiol. 57, 143-150.

Fries, P. (2005). A mechanism for cognitive dynamics: neuronal communication through neuronal coherence. Trends Cogn. Sci. (Regul. Ed.) 9, 474-480.

Gandour, J., Yunxia, T., Talavage, T., Wong, D., Dzemidzic, M., Yisheng, X., Xiaojian, L., and Lowe, M. (2007). Neural basis of first and second language processing of sentencelevel linguistic prosody. Hum. Brain Mapp. 28, 94-108.
Golestani, N., Price, C. J., and Scott, S. K. (2011). Born with and ear for dialects? Structural plasticity in the expert phonetician brain. J. $\mathrm{Neu}$ rosci. 31, 4213-4220.

Grabner, R. H., Neubauer, A. C., and Stern, E. (2006). Superior performance and neural efficiency: the impact of intelligence and expertise. Brain Res. Bull. 69, 422-439.

Hagoort, P., Hald, L., Bastiaansen, M. and Petersson, K. M. (2004). Integration of word meaning and world knowledge in language comprehension. Science 304, 438-441.

Haier, R., Siegel, B., Nuechterlein, K. Hazlett, E., Wu, J., Peak, J., Browning, H. L., and Buchsbaum, M. S. (1988). Cortical glucose metabolic rate correlates of abstract reasoning and attention studied with PET. Intelligence 11, 199-218.

Haier, R., Siegel, B., Tang, C. H., Abel, L., and Buchsbaum, M. (1992). Intelligence and changes in regional cerebral glucose metabolic rate following learning. Intelligence 16, 415-426.

Hald, L. A., Bastiaansen, M. C., and Hagoort, P. (2006). EEG theta and gamma responses to semantic violations in online sentence processing. Brain Lang. 96, 90-105.

Hasegawa, M., Carpenter, P. A., and Just, M. A. (2002). An fMRI study of bilingual sentence comprehension and workload. Neuroimage 15 647-660.

Herrmann, C., Munk, M., and Engel, A. (2004). Cognitive functions of gamma-band activity: memory match and utilization. Trends Cogn. Sci. (Regul. Ed.) 8, 347-355.

Hoke, M., Lehnertz, K., Pantev, C., and Lütkenhöner, B. (1989). "Spatiotemporal aspects of synergetic processes in the auditory cortex as revealed by magnetoencephalogram," in Series in Brain Dynamics, eds E. Basar and T. H. Bullock, (Berlin: Springer), 84-105.

Ihara, A., and Kakigi, R. (2006). Oscillatory activity in the occipitotemporal area related to the visual perception of letters of a first/second language and pseudoletters. Neuroimage 29 789-796.

Jasper, H. H. (1958). The ten/twenty electrode system of the international federation. Electroencephalogr. Clin. Neurophysiol. 10, 371-375.

Jausovec, N., and Jausovec, K. (2005). Differences in induced gamma and upper alpha oscillations in the human brain related to verbal/performance and emotional intelligence. Int. J. Psychophysiol. 56, 223-235.

Just, M. A., Carpenter, P. A., Keller, T. A., Eddy, W. F., and Thulborn, K. R.
(1996). Brain activation modulated by sentence comprehension. Science 274, 114-116.

Kaiser, J., and Lutzenberger, W. (2003). Induced gamma-band activity and human brain function. Neuroscientist 9, 475-484.

Klein, D., Watkins, K. E., Zatorre, R. J., and Milner, B. (2006). Word and nonword repetition in bilingual participants: a PET study. Hum. Brain Mapp. 27, 153-161.

Kotz, S. (2009). A critical review of ERP and fMRI evidence on L2 syntactic processing. Brain Lang. 109, 68-74.

Le Van Quyen, M., and Bragin, A. (2007). Analysis of dynamic brain oscillations: methodological advances. Trends Neurosci. 30, 365-373.

Leonard, M., Torres, C., Travis, K., Brown, T., Hagler, D., Dale, A., Elman, J., and Halgren, E. (2011). Language proficiency modulates the recruitment of non-classical language areas in bilinguals. PLoS ONE 6, e18240. doi:10.1371/journal.pone.0018240

Lotze, M., Scheler, G., Tan, H. R., Braun, C., and Birbaumer, N. (2003). The musician's brain: functional imaging of amateurs and professionals during performance and imagery. Neuroimage 20, 1817-1829.

Lucas, T. H., McKhann, G., and Ojemann, G. (2004). Functional separation of languages in the bilingual brain: a comparison of electrical stimulation language mapping in 25 bilingual patients and 117 monolingual control patients. J. Neurosurg. 101, 449-457.

Mac Whinney, B. (2010). "A tale of two paradigms," in Language Acquisition across Linguistic and Cognitive Systems, eds M. Kail and M. Hickmann (Amsterdam: John Benjamins), 17-33.

Majerus, S., Belayachi, S., De Smedt, B., Leclercq, A. L., Martinez, T., Schmidt, C., Weekes, B., and Maquet, P. (2008). Neural Networsk for short-term memory for order differentiate high and low proficiency bilinguals. Neuroimage 42, 1698-1713.

Marian, V., Spivey, M., and Hirsch, J. (2003).Shared and separate systems in bilingual language processing: converging evidence from eyetracking and brain imaging. Brain Lang. 86, 70-82.

Micheloyannis, S., Vourkas, M., Bizas, M., Simos, P., and Stam, C. J. (2003). Changes in linear and nonlinear EEG measures as a function of task complexity: evidence for local and distant signal synchronization. Brain Topogr. 15, 239-347. 
Mormann, F., Lehnertz, K., David, P., and Elger, C. E. (2000). Mean phase coherence as a measure for phase synchronization and its application to the EEG of epilepsy patients. Physica D 144, 358-369.

Newman-Norlund, R. D., Frey, S. H., Petitto, L. A., and Grafton, S. T. (2006). Anatomical substrates of visual and auditory miniature second-language learning. J. Cogn. Neurosci. 18, 1984-1997.

Nolte, G., Bai, O., Wheaton, L., Mari, Z., Vorbach, S., and Hallet, M. (2004). Identifying true brain interaction from EEG data using the imaginary part of coherency. Clin. Neurophysiol. 115, 2292-2307.

Ojima, S., Nakata, H., and Kakigi, R. (2005). An ERP study of second language learning after childhood: effects of proficiency. J. Cogn. Neurosci. 17, 1212-1228.

Oldfield, R. C. (1971). The assessment and analysis of handedness: the Edinburgh inventory. Neuropsychologia 9, 97-113.

Pakulak, E., and Neville, H. J. (2010). Proficiency differences in syntactic processing of monolingual native speakers indexed by event-related potentials. J. Cogn. Neurosci. 22, 2728-2744.

Perani, D., Dehaene, S., Grassi, F., Cohen, L., Cappa, S. F., Dupoux, E., Fazio, F., and Mehler, J. (1996). Brain processing of native and foreign languages. Neuroreport 7, 2439-2444.

Perani, D., Paulesu, E., Galles, N. S., Dupoux, E., Dehaene, S., Bettinardi, V., Cappa, S. F., Fazio, F., and Mehler, J. (1998). The bilingual brain. Proficiency and age of acquisition of the secon language. Brain 121, 1841-1852.

Pereda, E., Quian Quiroga, R., and Bhattacharya, J. (2005). Nonlinear multivariate analysis of neurophysiological signals. Prog. Neurobiol. 77, 1-37.

Pulvermüller, F., Birbaumer, N., Lutzenberger, W., and Mohr, B. (1997). High-frequency brain activity: its possible role in attention, perception and language processing. Prog. Neurobiol. 52, 427-445.
Raichle, M. E., Fiez, J. A., Videen, T. O., MacLeod, A. M., Pardo, J. V., Fox, P. T., and Petersen, S. E. (1994). Practice-related changes in human brain functional anatomy during nonmotor learning. Cereb. Cortex 4, 8-26.

Reiterer, S. (2010). "The cognitive neuroscience of second language acquisition and bilingualism: factors that matter in L2 acquisition - a neurocognitive perspective," in Language Acquisition across Linguistic and Cognitive Systems, eds M. Kail and M. Hickmann (Amsterdam: John Benjamins), 307-321.

Reiterer, S., Hemmelmann, C., Rappelsberger, P., and Berger, M. L. (2005a). Characteristic functional networks in high-versus low-proficiency second language speakers detected also during native language processing: an explorative EEG coherence study in 6 frequency bands. Brain Res. Cogn. Brain Res. 25, 566-578.

Reiterer, S., Berger, M. L., Hemmelmann, C., and Rappelsberger, P. (2005b). Decreased coherence between prefrontal electrodes: a correlate of high language proficiency? Exp. Brain Res. 163, 109-113.

Reiterer, S., Hu, X., Erb, M., Rota, G., Nardo, D., Grodd, W., Winkler, S., and Ackermann, H. (2011). Individual differences in speech imitation/pronunciation aptitude in late bilinguals: functional neuroimaging and brain morphology. Front. Psychol. 2:271, 1-12.

Reiterer, S., Pereda, E., and Bhattacharya, J. (2009). Measuring second language proficiency with EEG synchronization: how functional cortical networks and hemispheric involvement differ as a function of proficiency level in second language speakers. J. Sec. Lang. Res. 25, 77-106.

Rypma, B., and D’Esposito, M. (1999). The roles of prefrontal brain regions in components of working memory: effects of memory load and individual differences. Proc. Natl. Acad. Sci. U.S.A. 96, 6558-6563.

Sarnthein, J., Petsche, H., Rappelsberger, P., Shaw, G. L., and von Stein,
A. (1998). Synchronization between prefrontal and posterior association cortex during human working memory. Proc. Natl. Acad. Sci. U.S.A. 95, 7092-7096.

Sauseng, P., Klimesch, W., Schabus, M., and Doppelmayr, M. (2005). Fronto-parietal EEG coherence in theta and upper alpha reflect central executive functions of working memory. Int. J. Psychophysiol. 57, 97-103.

Schack, B., Klimesch, W., and Sauseng, P. (2005). Phase synchronization between theta and upper alpha oscillations in a working memory task. Int. J. Psychophysiol. 57, 105-114.

Simos, P. G., Papanikolaou, E., Sakkalis, E., and Micheloyannis, S. (2002). Modulation of gamma-band spectral power by cognitive task complexity. Brain Topogr. 14 191-196.

Stam, C. J. (2005). Nonlinear dynamical analysis of EEG and MEG: review of an emerging field. Clin. Neurophysiol. 116, 2266-2301.

Stam, C. J., Nolte, G., and Daffertshofer, A. (2007). Phase lag index: assessment of functional connectivity from multi channel EEG and MEG with diminished bias from common sources. Hum. Brain Mapp. 28 1178-1193.

Thompson-Schill, S. L., D’Esposito, M. and Kan, I. P. (1999). Effects of repetition and competition on activity in left prefrontal cortex during word generation. Neuron 23, 513-522.

Vingerhoets, G., Van Borsel, J., Tesink, C., Deblaere, K., Seurinck, R., Vandemaele, P., and Achten, E. (2003). Multilingualism: an fMRI study. Neuroimage 20, 2181-2196.

Ward, L. M. (2003). Synchronous neural oscillations and cognitive processes. Trends Cogn. Sci. (Regul. Ed.) 7, 553-559.

Wartenburger, I., Heereken, H., Abutalebi, J., Cappa, S., Villringer, A., and Perani, D. (2003). Early setting of grammatical processing in the bilingual brain. Neuron 37, 159-170.

Weiss, S., Mueller, H. M., Schack, B., King, J. W., Kutas, M., and Rappelsberger, P. (2005). Increased neuronal communication accompanying sentence comprehension. Int. J. Psychophysiol. 57, 129-141.

Wells, G. (1985). Language Development in the Pre-School Years. Cambridge: Cambridge University Press.

Xue, G., Dong, Q., Jin, Z., and Chen, C. (2004). Mapping of verbal working memory in nonfluent ChineseEnglish bilinguals with functional MRI. Neuroimage 22, 1-10.

Yetkin, O., Zerrin, T., Yetkin, F., Haughton, V., and Cox, R. W. (1996). Use of functional MR to map language in multilingual volunteers. AJNR Am. J. Neuroradiol. 17, 473-477.

Zhang, Y., Kuhl, P. K., Imada, T. Kotani, M., and Tohkura, Y. (2005). Effects of language experience: neural commitment to languagespecific auditory patterns. Neuroimage 26, 703-720.

Conflict of Interest Statement: The authors declare that the research was conducted in the absence of any commercial or financial relationships that could be construed as a potential conflict of interest.

Received: 30 April 2011; paper pending published: 11 June 2011; accepted: 29 October 2011; published online: 22 November 2011.

Citation: Reiterer S, Pereda $E$ and Bhattacharya J (2011) On a possible relationship between linguistic expertise and EEG gamma band phase synchrony. Front. Psychology 2:334. doi: 10.3389/fpsyg.2011.00334

This article was submitted to Frontiers in Cognition, a specialty of Frontiers in Psychology.

Copyright (c) 2011 Reiterer, Pereda and Bhattacharya. This is an open-access article subject to a non-exclusive license between the authors and Frontiers Media $S A$, which permits use, distribution and reproduction in other forums, provided the original authors and source are credited and other Frontiers conditions are complied with. 